\title{
Astrophysics research at DAP
}

\author{
P.N. Diep*, D.T. Hoai, P.T. Nhung, N.T. Phuong, T.T. Thai, P. Tuan-Anh and P. Darriulat \\ Department of Astrophysics, Vietnam National Space Center, Vietnam Academy of Science and \\ Technology (VNSC/VAST), 18 Hoang Quoc Viet, Nghia Do, Cau Giay, Hanoi, Vietnam
}

\begin{abstract}
The research and training team of the Department of Astrophysics (DAP), Vietnam National Space Center, is some twenty years old. Currently, we work in the fields of stellar physics and high-redshift galaxies using $\mathrm{mm} / \mathrm{sub}-\mathrm{mm}$ data observed by world-leading radio interferometers such as ALMA and NOEMA. In this report, we present the research team and its activities and give illustrations of the research work with emphasis on the use of archival data.
\end{abstract}

\section{Introduction}

DAP, formerly Vietnam Astrophysics Training Laboratory (VATLY), hosts a research team that was put together twenty years ago by Professor Pierre Darriulat at the Institute for Nuclear Science and Technology in Hanoi. Formerly a particle physicist and Research Director of CERN (European Organization for Nuclear Research) he brought to Vietnam detectors and fast electronics units that had been used in particle physics experiments at CERN and had been given to him to help with the development of scientific research in the country. These instruments were old but still working well. In the early years of the laboratory, we used them to set up experiments by ourselves to study cosmic rays which come from space free of charge. By chance, Hanoi sits on the Earth magnetic equator, meaning maximal deflection and therefore higher energy for charged cosmic ray particles. The effect is measured by the so-called rigidity cut-off which is $17 \mathrm{GV}$ in Hanoi, $11 \mathrm{GV}$ in Japan and only $4 \mathrm{GV}$ in Europe and North America. One of our highlight experiments was a series of three measurements of the atmospheric muon flux in Hanoi using a movable home-made telescope. These contributed useful information for neutrino oscillation experiments such as Super-Kamiokande that need to map the muon flux on the Earth surface in order to model what they expect to detect. Indeed both atmospheric muons and neutrinos are decay products of pions in the cascade of secondary particles produced by primary ultra-high energy cosmic rays coming from outer space: muons are therefore tracers of neutrinos. We provided physicists from Super-Kamiokande with our measurements and found them in good agreement with the predictions of their model $[1,2]$. Besides carrying experiments by ourselves we also joined the collaboration of the Pierre Auger Observatory (PAO) located in Malargue, in the Argentinean pampa, the largest international collaboration dedicated to the study of extreme energy (extra-galactic) cosmic rays. We took part in many activities of the collaboration: spending time in Malargue to setup and maintain detectors, to take shifts, to attend collaboration meetings, and most

\footnotetext{
* Corresponding author: pndiep@,vnsc.org.vn
} 
importantly to analyse PAO data. Three of us got $\mathrm{PhD}$ degrees making our thesis work in the framework of this collaboration. In Hanoi, on the roof of our laboratory, we built a model of the Cherenkov counter of which the PAO array is made, with 1600 such units covering an area of $3000 \mathrm{~km}^{2}$.

We progressively built up a keen interest for astrophysics. Moreover, as working in a big collaboration was making it difficult for the team to develop an identity of its own, we gradually shifted our interest to radio astronomy where front-line research could be made on studies matching well the size and expertise of a small team. With radio astronomy, the young members of the team were first trained in the laboratory using a pair of Yagi antennas coupled in interferometer mode, a gift of Professor Nguyen Quang Rieu from Paris Observatory. It was used to observe solar interferences and made the subject of a master thesis. Then Pierre bought for us a $2.6 \mathrm{~m}$ diameter telescope tuned on the HI line with which we observed the Milky Way, the Sun, the Moon and a few other bright radio sources. It produced nine articles in refereed journals, two master theses and two bachelor dissertations. At the same time we started collaborating with French astrophysicists in Toulouse and Paris on jointly supervised PhD research works. Such collaboration, called "cotutelle" in French, means that for three successive years the PhD student spends part of the year with the Vietnamese team and the other part with the team of the French cosupervisor. The thesis is written in English with substantial summaries in Vietnamese and French, the defence takes place in one of the two countries with a mixed panel and cochairs from each country and the successful student is awarded both a Vietnamese and a French degree. We were familiar with the scheme for having made use of it three times already with the PAO collaboration. The team includes now three members who obtained their $\mathrm{PhD}$ degrees in a cotutelle between a Vietnamese doctoral school and Toulouse University, Paris Observatory and University of Bordeaux. The subjects are in the fields of high red-shift galaxies, evolved stars and star formation respectively. This way of supervising students is truly beneficial both for the $\mathrm{PhD}$ students and the whole team, in particular for a young research team like ours. In addition it makes the seed for possible future collaborations.

In January 2015 the whole team moved to the Vietnam National Space Center, which is one of some thirty institutions of the Vietnam Academy of Science and Technology, and became its Department of Astrophysics (DAP).

Currently, astronomy is not included in the curriculum of Vietnamese universities. Only a few universities offer introductory lectures, usually within the frame of the physics departments; an exception is the new University of Science and Technology of Hanoi (USTH) where students can choose to major in astrophysics. We participate in the process of building the teaching programmes in astronomy at USTH, giving lectures, practical classes, and supervising USTH students for their theses and internships at both bachelor and master levels. One of the USTH former master students is staying with us. After having spent six months in France working on MESSEGER data exploring the polar region of planet Mercury for her master thesis, she joined us and we helped her with the completion of the analysis and the redaction of the journal article.

Vietnam recently joined the East Asian Observatory (EAO) as observer. This gave several members of the team the opportunity to join observation programmes of the James Clerk Maxwell Telescope (JCMT) such as a study of magnetic fields in star forming regions (BISTRO) or a survey of nearby evolved stars (NESS). We are taking an active part in the efforts deployed by SEAAN and EAO to foster collaboration in the region, to enhance the quality and quantity of the research work, and to boost training in astronomy and astrophysics.

In Vietnam we devote time and effort to outreach activities aimed at fostering the interest of the public in astronomy and space sciences. In particular we help with the 
exploitation of VNSC facilities in Hoa Lac, near Hanoi, hosting a 100-seat planetarium, a $50 \mathrm{~cm}$ optical telescope and a science museum under current completion. We also take part in training high school students to participate in the International Astronomy and Astrophysics Olympiads (IOAA) and generally in various activities related to public education and training.

Over the past 18 years we have trained 8 PhDs, 10 masters, and 12 bachelor students. We also hosted many interns from both inside Vietnam and abroad. We are the co-authors of 50 SCI articles with the Pierre Auger Collaboration and have published $30 \mathrm{SCI}$ and 31 ASEAN Citation Index articles and conference proceedings of our own. By the end of 2019, the team included ten members (Fig. 1). We publish a Newsletter twice a year where we report on the life of the laboratory and on our research and training activities. More information is available in our web site: https://dap.vnsc.org.vn/.

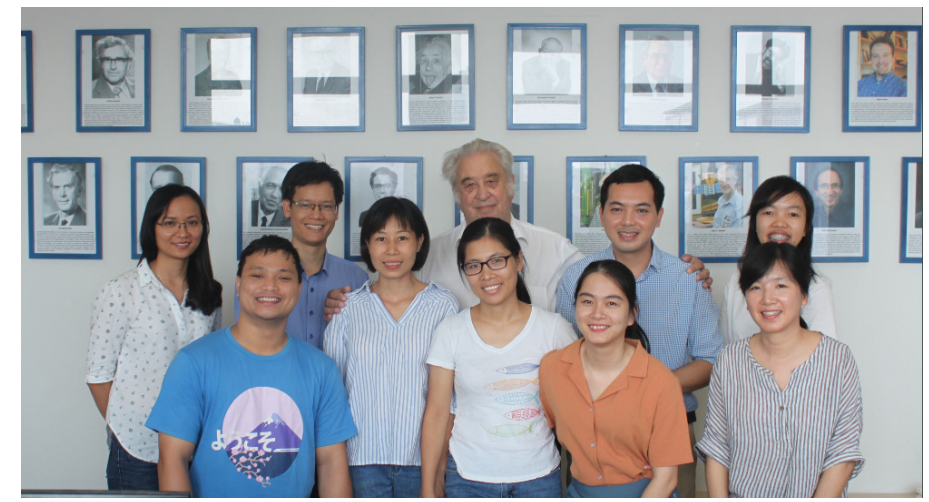

Fig. 1 The DAP team (October 2019).

\section{Research at DAP}

Currently, we focus on studies of stellar physics and of far-away galaxies that are observed as they were many Gyr ago. The data which we use are mostly from radio interferometers such as ALMA and NOEMA, the two largest arrays in the southern and northern hemispheres respectively. ALMA stands for Atacama Large Mm/sub-mm Array and NOEMA for NOthern Extended Millimeter Array. ALMA is located in the Atacama desert of the Chilean Andes at 5'000 m above sea level. It consists of 66 antennas of two types, 12 and 7 meters in diameter. NOEMA is in the French Alps on the Plateau de Bure at an altitude of 2'550 m. The complete configuration of NOEMA will include 12 antennas of 15 $\mathrm{m}$ diameter by the end of 2021. The signals detected by the antennas of an interferometer array are combined to provide high angular resolution observations of astronomical sources.

Vietnam is not a member of the ALMA and NOEMA partnerships. However, these partnerships, like is often the case in basic research, have a very generous policy aimed in particular at fostering interest for science in developing countries. ALMA make their observations openly accessible to public after a proprietary time of only one year and we are making intensive use of this opportunity. The quality of the data is usually excellent and the quantity available is huge. This open access policy is truly vital for our survival, in particular since our country gives priority to applied rather than basic research and our authorities cannot afford paying important membership fees to international collaborations. As mentioned earlier, we pay much attention to maintaining fruitful contacts with colleagues abroad: such collaboration gives us opportunities not only to enhance our 
knowledge and skills but also to join them in submitting proposals for observations using facilities to which they have access. Moreover EAO is operating the James Clerk Maxwell Telescope and will take over more telescopes from the region in the future. This gives us access to all the facilities of the Observatory.

A few illustrations of the research work that we are doing are presented below.

\subsection{High redshift galaxies}

The study of high redshift (high z) galaxies opens a window on the early Universe: they are observed in the state in which they were several Gyr ago. Their study provides important constraints on the mechanism of galaxy formation and insights into physical processes such as star formation in the early Universe. A major difficulty is that such galaxies are very far away and, therefore, very faint. However, this is helped by gravitational lensing: if there is a massive object on the line of sight between the observer and the source, it acts as a lens by bending light rays and an important magnification may result. The mass acting as a lens is usually a galaxy or a cluster of galaxies. The price to pay is that the images are strongly distorted, making the reconstruction of the source brightness distribution difficult. When the lens is essentially a single galaxy one speaks of strong lensing, the configuration that applies to most cases of far-away galaxies that have been studied (see e.g. Blandford et al. [3] or Saha and Williams [4]). We studied in great detail a gravitationally lensed galaxy that hosts a quasar in its centre, RX J0911, at z 2.8, meaning looking back 11.3 Gyr. It is lensed by a foreground galaxy into four images that have been observed by the HST. We studied observations of its gas and dust content, the mm emission of which extends over a large region with very different imaging properties depending on their projected distance to the lens. The problem of de-lensing these observations is difficult and requires, in addition to the knowledge of the quasar HST images, a model of the lens mass distribution [5]. We studied the emission of the $\mathrm{CO}(7-6)$ line measured by NOEMA and the continuum emission measured by ALMA. Morpho-kinematics properties of the gas [6] and of the dust [7] have been deduced and possible physical interpretations have been discussed [4]. The gas extends over a distance of $850 \pm 120 \mathrm{pc}$ from the centre of the galaxy and its distribution gives evidence for significant ellipticity and velocity gradient caused by molecular outflow and rotation (Fig. 2). The dust component is more compact, by a factor of $\sim 3.4 \pm 0.4$. These observations make RX J0911 one of the few high-z galaxies for which both dust and gas have been resolved and measured.
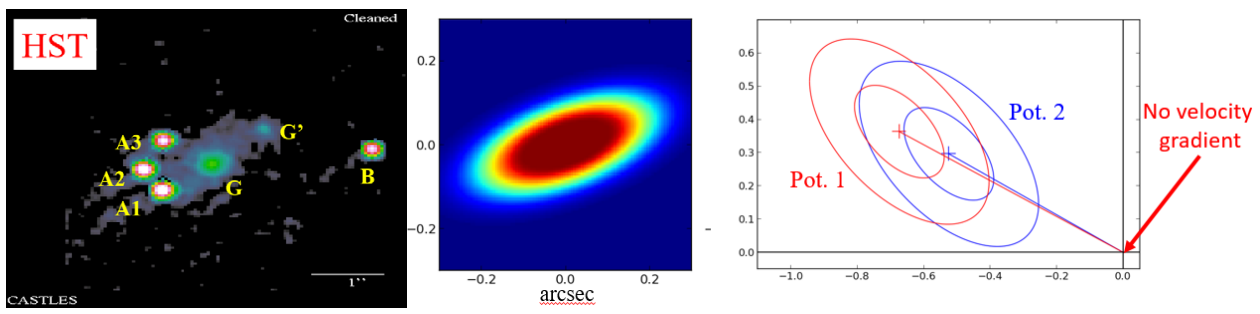

Fig. 2 Left: RX J0911 observed by the Huble Space Telescope. Centre: the gas component revealing ellipticity. Right: Evidence for rotation. 


\subsection{Evolved stars}

The study of evolved star aims at understanding how a star such as the Sun evolves at the end of its lifetime from a nearly perfect spherical shape to the diversity of configurations observed in planetary nebulae. Which are the most important parameters governing the symmetry breaking? Evolved stars often feature a bipolar molecular outflow. A number of evolved stars at different stages of evolution and different orientations in space have been studied at DAP using observations of the high resolution continuum and line emissions from dust grains and gas molecules [8-15]. Methods to study and reconstruct in space axisymmetric radio sources (a common feature of young and old stars) have been developed to this effect $[16,17]$. A review of studies made by the team in this field, dealing with the presence of high Doppler velocity wings in the spectra of O-rich AGB stars, is presented elsewhere in these Proceedings [18].

Here we show as an example a study of the Red Rectangle using ALMA archival data. It was one of the first stars that we studied after having switched from cosmic rays to radio astronomy [19]. The Red Rectangle is a post-AGB star having its axis perpendicular to the line of sight. It looks like a red rectangle when observed in infrared hence the name. AGB stands for Asymptotic Giant Branch which is a region of the Hertzsprung-Russell diagram populated by cool luminous Red Giants. All low- to intermediate-mass stars ( 0.6 to 10 solar masses) will go through this phase of stellar evolution. Post-AGB stars are on the way to become planetary nebulae having a degenerate core that will soon be a White Dwarf when what is left of the circumstellar envelope will have completely diluted in the interstellar medium. For the study of the Red Rectangle we used ALMA observations of the $\mathrm{CO}(6-5)$ and $\mathrm{CO}(3-2)$ emissions of unprecedented quality.
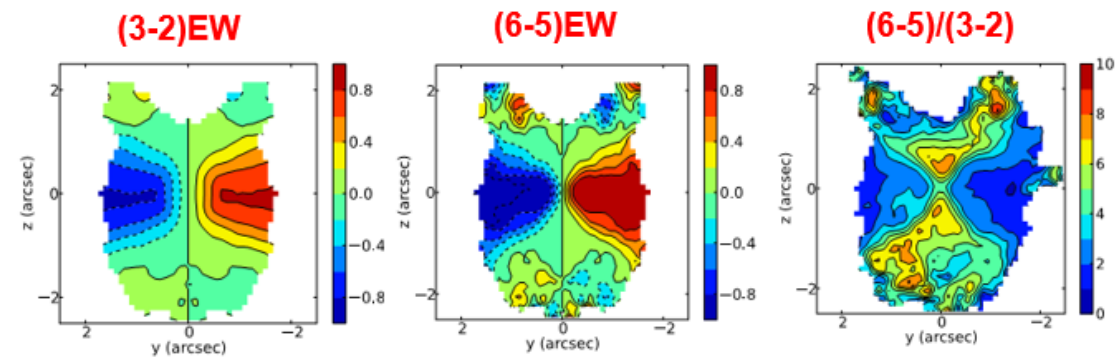

Fig. 3 Red Rectangle: mean Doppler velocity maps (left and centre) and line intensity ratio (right).

Fig. 3 (left and centre) displays the mean Doppler velocity maps for each line separately. The East-West asymmetry reveals clearly the rotation of the equatorial region about the star axis. The map of the ratio of the two line intensities, a proxy of temperature, shows evidence for a biconical structure down to very small distances from the central star (Fig. 3 right). We modelled the gas kinematics dividing the velocity fields in two regions. In the polar regions, the lines of field are meridian parabolas joining smoothly with the equatorial region where trajectories are spirals accounting for both rotation and expansion (Fig. 4 left and central). The best fit (Fig. 4 right) gives a surprisingly good description of the data given the crudeness of the model. 


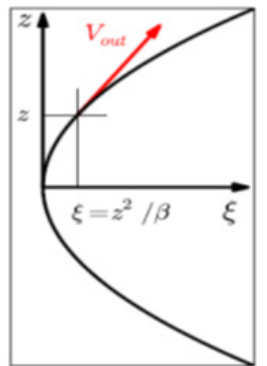

Polar region

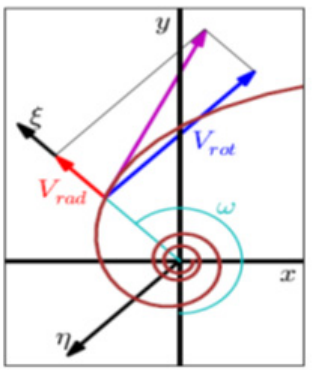

Equatorial region

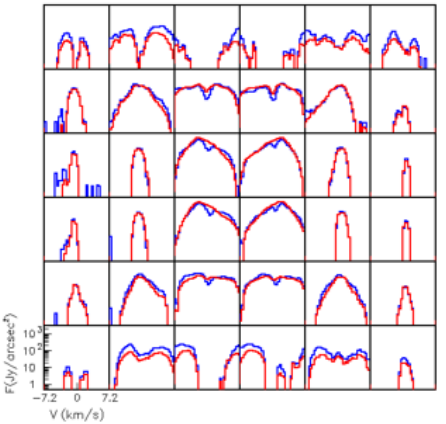

Fig. 4 Red Rectangle. Left and centre: gas velocity and trajectories. Right: Doppler velocity spectra comparing data (blue) and model (red).

The rotation and radial velocities are found equal to $1.0 \mathrm{~km} / \mathrm{s}$ and $1.6 \mathrm{~km} / \mathrm{s}$ respectively. Using the velocity field model and the comparison between the two line emissions we have been able to reconstruct the spatial distributions of both temperature and density, displaying a sharp separation between equatorial and polar regions (Fig. 5).

Temperature

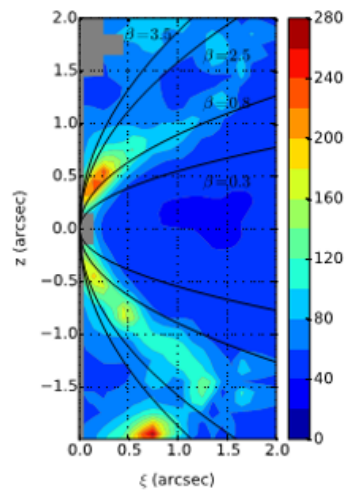

Density $\times r^{2}$

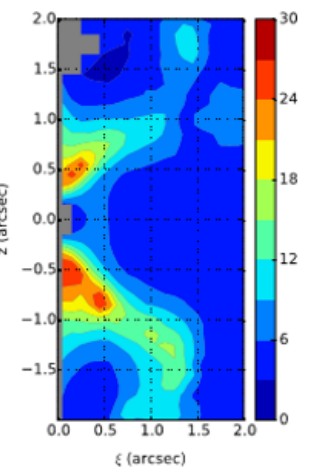

Fig. 5 Meridian distributions of temperature (left) and density multiplied by the square of the distance to the central star (right).

\subsection{Young stars}

Young stars form from the condensation of gas and dust in a molecular cloud. In-falling matter toward the centre of the condensed core forms rapidly an accretion disc. The cool dust accumulates in the disc plane onto which gas molecules freeze out, forming an ice mantel. Some dust grains aggregate to form larger and larger bodies, ultimately planetesimals and planets. A good summary of the current research in the field can be found in a review by Sean Andrews [20]. A topic of strong current interest is the formation of planets in such discs, called for this reason protoplanetary discs. In particular one aims at identifying clearly which physico-chemical conditions need to be fulfilled for planets to start forming. We have studied several young stars [21-23], in particular a multiple stellar system, GG Tau A, which is presented below.

GG Tau A, located at $150 \mathrm{pc}$ in the Taurus molecular cloud [24,25], is considered to be an archetype of a T-Tauri - solar mass protostar- multiple system. It is a triple star made of 
GG Tau Aa and a nearby binary GG Tau Ab1/Ab2 with respective separations of 35 au and $4.5 \mathrm{au}$. GG Tau A is surrounded by a circumbinary (referring to Aa and $\mathrm{Ab}$ ) envelope of gas and dust in the form of a ring spanning from $\sim 190$ to 280 au in distance from GG Tau A and an outer disc extending up to $\sim 800$ au with a total mass $\sim 0.15$ solar masses. Using most of the available archival and new data from ALMA and NOEMA, in collaboration with experienced colleagues from University of Bordeaux, we studied the morphology, kinematics, and physical conditions of the gas and dust (distribution of temperature and density) [26,27]. The high sensitivity of the observations made it possible to explore the properties of the cavity contained inside the central ring, at distances smaller than $\sim 140$ au from the central stars, where gas is falling along two streamers onto the circumstellar discs. A preliminary study of the chemistry at stake has revealed the presence of $\mathrm{H}_{2} \mathrm{~S}$; this was the first example of detection of the molecule in this type of protostars, made possible thanks to the relatively high mass of the GG Tau A disc [28].
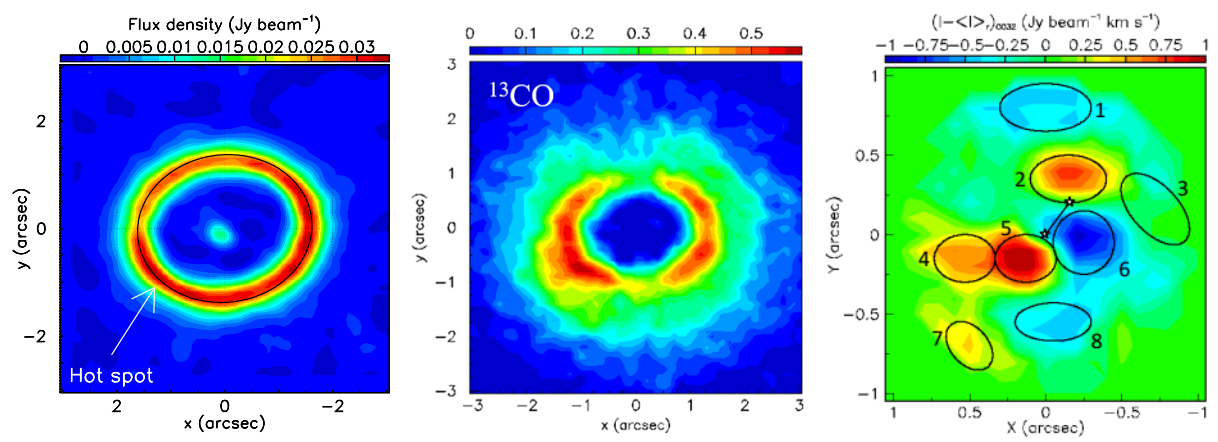

Fig. 5 GG Tau A. Left: intensity map of the dust ring. Centre: ${ }^{13} \mathrm{CO}$ gas distribution around the cavity. Right: emission in the cavity revealing the presence of gas streams.

\section{Conclusions}

Astrophysics, one of the most dynamic branches of modern physics, matches well the needs of a team such as ours. We have made significant contributions to the study of high redshift galaxies and a number of evolved stars and protostars.

Our experience shows that both training at home and collaboration with experienced teams abroad are mandatory for doing good research and making progress.

We are making extensive use of the archival data of the ALMA collaboration. Open access policy is a precious opportunity for astronomers, in particular in developing countries. Nowadays, most of the major observatories open their data to public: ALMA, NOEMA, and many facilities of ESO and NASA. They usually have very good supporting interface for searching, calibrating, and reducing data. Many of the data are already calibrated and ready to be used. We are all invited to use this treasure. As Pierre uses to say,

The sky belongs to all of us

We are all made of the same star dust.

\section{Acknowledgements}

All along the development of the team we have received invaluable help and support from many friends from abroad: Jim Cronin, Alan Watson, Alain Cordier, Pierre Billoir and Murat Boratav in the PAO collaboration; our present collaborators Thibaut Le Bertre, Anne Dutrey, Stéphane Guilloteau, Pierre Lesaffre and Jan Martin Winters; Antonino Zichichi and the World Association of Scientists who place in us unconditional confidence and give 
us continuous support; Jean Tran Thanh Van and the ICISE centre in Quy Nhon who help Vietnam with its progress in developing basic research; Nguyen Quang Rieu, whom we consider as the founding father of the Vietnamese radio astronomy and gave us advice and support in the early phase of our development; Paul Ho from EAO, Young-Chol Minh from KASI, Kazuhiro Sechiguchi from NAOJ and Boonrucksar Soonthornthum from NARIT, who give us constant support and help us integrating the regional activity and contributing to its progress; in Vietnam, we had the honour to count among our friends Hoang Tuy and Viet Phuong, who are no longer with us, and today scientists such as Pham Duy Hien and Nguyen Duc Chien who follow our progress and are always prepared to give us help and advice. To all we express our deepest gratitude from the bottom of our heart.

We are deeply indebted to the ALMA partnership, whose open access policy means invaluable support and encouragement for Vietnamese astrophysics. ALMA is a partnership of ESO (representing its member states), NSF (USA), NINS (Japan), NRC (Canada), NSC/ASIAA (Taiwan), and KASI (South Korea), in cooperation with Chile. The Joint ALMA Observatory is operated by ESO, AUI/NRAO and NAOJ. Financial support from the World Laboratory, Rencontres du Viet Nam, the Odon Vallet fellowships is gratefully acknowledged. Our research is funded by Vietnam National Foundation for Science and Technology Development (NAFOSTED) under grant number 103.99-2018.325.

\section{References}

1. P.N. Dinh, N.T. Dung, B.D. Hieu, et al., Nuclear Physics B, 661, 302 (2003)

2. P.N. Diep, P.N. Dinh, N.H. Duong, et al., Nuclear Physics B, 678, 3 (2004)

3. R.D. Blandford, et al., Science, 245, 824 (1989)

4. P. Saha \& L.L.R. Williams, AJ, 125, 2769 (2003)

5. D.T. Hoai, D.T. Hoai, P.Tuan-Anh, et al., RAA, 13, 803 (2013)

6. P. Tuan-Anh, F. Boone, D.T. Hoai, et al, A\&A, 552, L12 (2013)

7. P. Tuan-Anh, D.T. Hoai, D.T. Hoai, et al., MNRAS, 467 (3), 3513-3524 (2017)

8. D.T. Hoai, L. Mathews, J.M. Winters, et al., A\&A, 565, A54 (2014)

9. P.T. Nhung, D.T. Hoai, J.M. Winters, et al., RAA, 15, 713 (2015)

10. D.T. Hoai, P.N. Diep, N.T. Phuong, et al., RAA, 16, 111, (2016) arXiv:1601.04834

11. D.T. Hoai, P.T. Nhung, P.N. Diep, et al., RAA, 2016, 16, 106, arXiv:1601.01439

12. P.T. Nhung, D.T. Hoai, J.M. Winters et al., A\&A, 583, A64 (2015)

13. P.T. Nhung, P. Tuan-Anh, T. Le Bertre, et al., RAA, 19, 43 (2019)

14. D.T. Hoai, P.T. Nhung, P. Tuan-Anh, et al., MNRAS, 484, 1865 (2019)

15. P. Tuan-Anh, D.T. Hoai, P.T. Nhung, et al., MNRAS, 487, 622 (2019)

16. P.N. Diep, N.T. Phuong, D.T. Hoai, et al., MNRAS 461, 4276 (2016)

17. P.T. Nhung, D.T. Hoai, P. Tuan-Anh, et al., MNRAS, 480, 3324 (2018)

18. D.T. Hoai, P.T. Nhung, P. Tuan-Anh, et al., Proceedings of 11 th SEAAN, to be published, (2020)

19. P. Tuan-Anh, P.N. Diep, P.T. Nhung, et al., RAA, 12, 2213 (2015)

20. S. Andrews, ARAA, arXiv: 2001.05007, to be published (2020)

21. P.T. Nhung, D.T. Hoai, P. Tuan-Anh, et al., MNRAS, 469, 4726 (2017)

22. P. Tuan-Anh, P.T. Nhung, D.T. Hoai, et al., MNRAS, 463, 3563 (2016)

23. P.N. Diep, D.T. Hoai, N.B. Ngoc et al., VJSTE, Vol. 61, No. 2, p. 3-16 (2020) 
24. Gaia Collaboration, A. G. A. Brown, A. Vallenari, et al., "ArXiv e-prints" arXiv: 1804.09365 (2018)

25. Gaia Collaboration, T. Prusti, J. H. J. de Bruijne, et al., A\&A, 595, A1 (2016)

26. N.T. Phuong, P.N. Diep, A. Dutrey, et al., RAA, 18, 3, arXiv:1801.00861 (2018)

27. N.T. Phuong, A. Dutrey, P.N. Diep, et al., A\&A, to be published, (2020)

28. N.T. Phuong, E. Chapillon, L Majumdar, et al., A\&A, 616, L5 (2018) 